\section{First Human Case of Metacestode Infection Caused by Versteria sp. in a Kidney Transplant Recipient}

\begin{abstract}
Sapha Barkati, ${ }^{1,2, \Theta}$ Bruno Gottstein, ${ }^{3}$ Norbert Müller, ${ }^{3}$ Caroline Sheitoyan-Pesant, ${ }^{4}$ Peter Metrakos, ${ }^{2}$ Tianyan Chen, ${ }^{2}$ Richard Garceau, ${ }^{4}$ Michael D. Libman, ${ }^{1,2}$ Momar Ndao, ${ }^{1,5}$ and Cedric P. Yansouni ${ }^{1,2}$

1 J.D. MacLean Centre for Tropical Diseases at McGill University, and ${ }^{2}$ McGill University Health Centre, Montreal, Quebec, Canada; ${ }^{3}$ nstitute of Parasitology, Vertsuisse Faculty and Faculty of Medicine, University of Bern, Switzerland; and ${ }^{4}$ Centre Hospitalier Universitaire Dr-Georges-L.-Dumont, Moncton, New-Brunswick, and ${ }^{5}$ National Reference Centre for Parasitology, Research Institute of the McGill University Health Centre, Montreal, Quebec, Canada
\end{abstract}

Cestodes are emerging agents of severe opportunistic infections among immunocompromised patients. We describe the first case of human infection, with the recently-proposed genus Versteria causing an invasive, tumor-like hepatic infection with regional and distant extension in a 53-year-old female kidney transplant recipient from Atlantic Canada.

Keywords. metacestode infection; Versteria; kidney transplant recipient; immunosuppression; zoonosis.

A 53-year-old Caucasian female from rural New Brunswick (Atlantic Canada) presented to a community hospital in April 2017 with a 3-day history of fever, productive cough, myalgia, malaise, and anorexia. Her past medical history was remarkable for obstructive nephropathy necessitating a kidney transplant in 1992, for which she was on minimal immunosuppression with tacrolimus, mycophenolate mofetil, and prednisone (5 mg thrice weekly). Physical examination revealed a temperature of $40^{\circ} \mathrm{C}$, oxygen saturation of $93 \%$ on $2 \mathrm{~L} / \mathrm{min}$, hypotension, and tachycardia. The rest of the physical exam was noted as unremarkable. Laboratory findings on admission were notable for mild pancytopenia, decreased kidney function compared to baseline (estimated $\mathrm{CrCl} 22 \mathrm{~mL} / \mathrm{min}$ ), transaminitis ( 8X normal), and elevated lactate dehydrogenase (Supplementary Table 1). A chest X-ray revealed bibasilar alveolar opacities as more prominent in the right lung. Empiric antibacterial therapy was initiated for presumed community-acquired pneumonia and the patient was transferred to a tertiary care centre for further evaluation.

Received 21 May 2018; editorial decision 16 July 2018; accepted 25 July 2018; published online September 13, 2018.

Correspondence: S. Barkati, J. D. MacLean Centre for Tropical Diseases at McGill University, 1001 Boulevard Decarie, Montreal, Quebec H4A 3J1, Canada (sapha.barkati@mail.mcgill.ca).

\section{Clinical Infectious Diseases ${ }^{\circledR} \quad 2018 ; X X(X X): 1-4$}

(C) The Author(s) 2018. Published by Oxford University Press for the Infectious Diseases Society of America. All rights reserved. For permissions, e-mail: journals.permissions@oup.com. DOI: $10.1093 /$ cid/ciy602
Despite empiric meropenem, recurrent episodes of hypotension required vasopressors and repeated admissions to the intensive care unit. A chest computed tomography (CT) scan showed mixed alveolar ground glass opacities and a prominent right hilar lymph node $(3.9 \times 2 \mathrm{~cm})$. Abdominal imaging revealed a large heterogeneous central hepatic lesion $(19.3 \times 15 \times 8.7 \mathrm{~cm})$ abutting the middle hepatic and left portal veins, with multiple satellite nodules (Figures 1 and 2). An abdominal CT scan performed 3 years earlier was unremarkable. Further tests-including a cerebral CT scan, a transesophageal echocardiogram, and a colonoscopy-revealed no other lesions. A laparoscopic liver biopsy revealed extensive necrosis, with focal necrotizing granulomas surrounding hooklets and a single protoscolex, consistent with a metacestode infection (Figure 3). Empiric treatment with albendazole for possible alveolar echinococcosis (AE) was initiated, and the patient was transferred to the J.D. MacLean Centre for Tropical Diseases in Montreal for further management.

Notable exposure history included living near a forest with her dog and with peridomestic wildlife such as foxes, coyotes, moose, deer, small rodents, and mustelids. She enjoys gardening and blueberry picking and drinks water from a surface well. She never traveled outside Canada, and noted a single 4-day trip to Edmonton (Canada) in 1980.

A whole-body positron emission tomography scan with 2-deoxy-2-[fluorine-18] fluoro-D-glucose integrated with CT (WB 18F-FDG PET/CT Scan) demonstrated low cervical, mediastinal, bilateral hilar, right axillary, and periportal adenopathy, as well as intense heterogeneous uptake of FDG in the liver. Histopathology of a biopsy of the right hilar lymph node performed by endobronchial ultrasound revealed granulomatous inflammation with extensive necrosis. Bacterial, mycobacterial, and fungal cultures were negative. The hepatic lesion was not considered resectable.

A screening enzyme-linked immunoassay (ELISA) using in-house crude antigens for Echinococcus granulosus was positive, with an optical density of 0.60 (cut-off value: 0.35 ). A second ELISA using an E. granulosis hydatid fluid antigen (EgHF) as previously described was also positive (156 AU/ml; cut-off $\geq 1 \mathrm{AU} / \mathrm{ml}$ ) [1]. For E. multilocularis, 2 separate ELISAs, using (1) purified Em2 antigen (localized in the laminated layer) or (2) recombinant Em18 antigen (previously known as EmII/310 -antigen, localized in the tegument of the protoscolex and the germinal layer) were negative $[2,3]$. Confirmatory Western blots for E. granulosus and E. multilocularis were performed as previously described, and were negative as well $[1,4]$.

Needle biopsies of the liver were repeated and sent for molecular testing at the University of Bern, Switzerland. Deoxyribonucleic acid (DNA) was extracted from a fresh-frozen native biopsy 


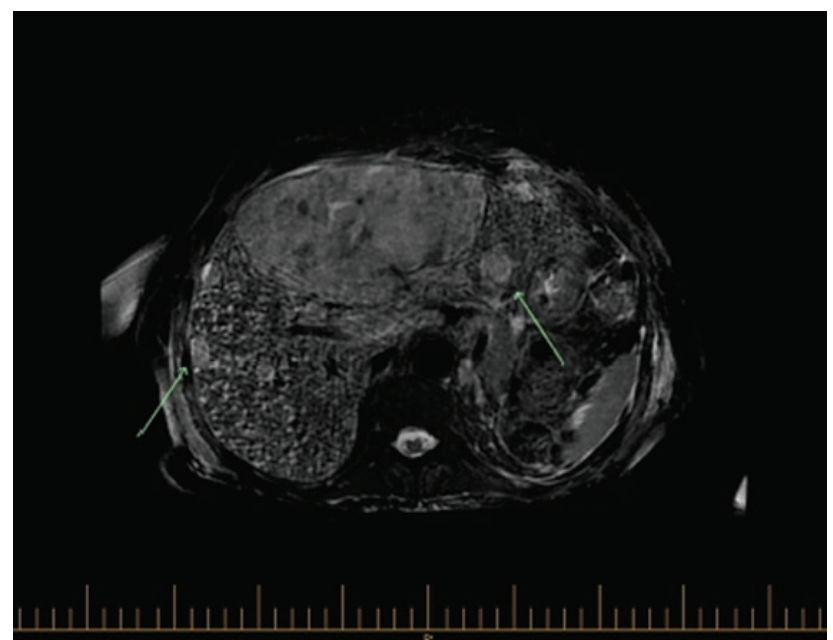

Figure 1. Axial T2-weighted magnetic resonance imaging scan of the liver, showing a large lesion occupying segments 3 and 4, with multiple satellite lesions (arrows), before initiation of albendazole and praziquantel.

specimen using a commercial kit, according to the manufacturer's instructions (QiAmp DNA mini kit, Qiagen, Hilden, Germany). In addition, DNA was also prepared from formalin-fixed paraffin-embedded liver tissue, as described by Müller et al. [5]. Multiplex polymerase chain reaction (PCR) targeting mitochondrial 12S rDNA for discrimination between different cestodes was performed as previously described [6]. E. granulosus and E. multilocularis can be identified directly, based on the size of their PCR product. A 264 base pair (bp) fragment was amplified from both fresh-frozen and paraffin-embedded specimens, corresponding to neither E. granulosus nor E. multilocularis. DNA sequencing of the PCR product was thus performed to identify the cestode.

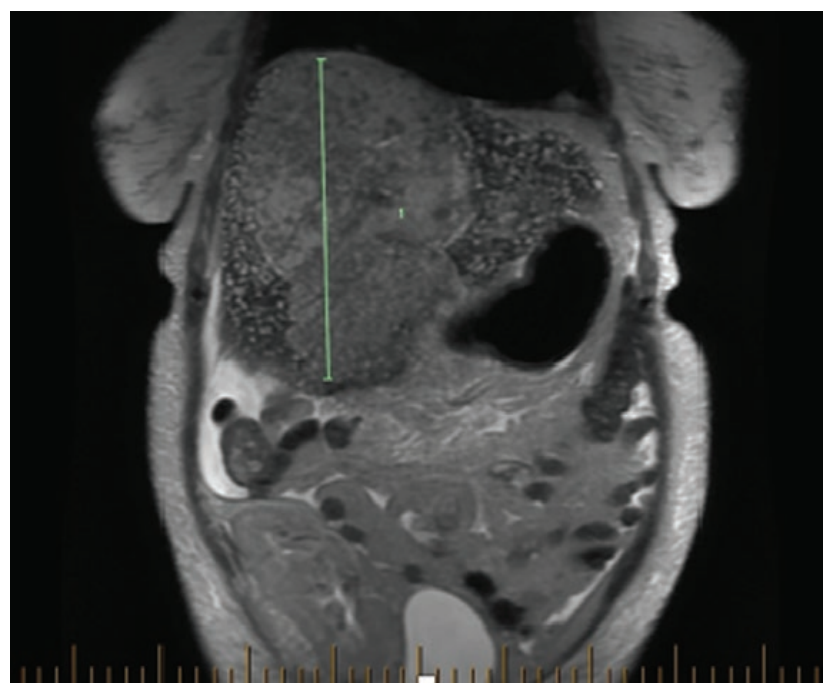

Figure 2. Coronal T2-weighted magnetic resonance imaging scan of the liver, showing a large lesion occupying segments 3 and 4, with multiple satellite lesions, before initiation of albendazole and praziquantel

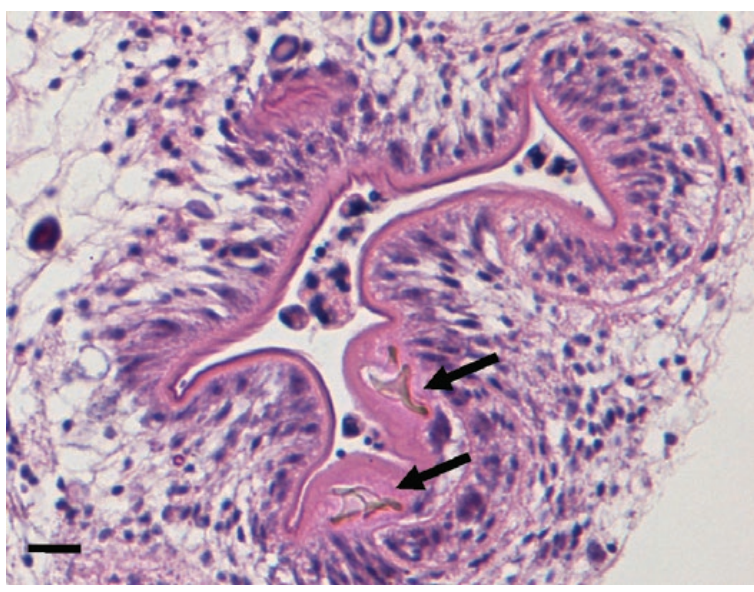

Figure 3. Hematoxylin and eosin staining of histopathology section, showing partial section through the parasite larvae with parts of a rostellum and a parenchymatous portion, plus 2 refractile hooklets (arrows). Scale bar represents $10 \mu \mathrm{m}$

Primers CEST3 and CEST5 were used for bidirectional sequencing of the PCR amplification product, as previously described [6]. Amplicons were purified using the Qiagen PCR purification kit (Qiagen, Hilden, Germany) and sequenced using the Microsynth DNA sequencing service (Balgach, Switzerland). Basic Local Alignment Search Tool (BLAST) analysis yielded 98\% identity with a sequence from a single reported case in a hominid: a fatal metacestode infection in a captive orangutan (Versteria sp., orangutan, Wisconsin; GenBank accession number: KF303341) [7]. Sequence data from the current case were deposited at GenBank (GenBank accession number: MH299497).

Striking similarities in the clinical, radiological, and histopathological features of this case with those of AE, and the high genetic relatedness of Versteria and Echinococcus, prompted us to manage our patient based on AE literature [8]. Antiparasitic therapy, combined with surgery, are the cornerstones of management. Our patient was treated with albendazole $(15 \mathrm{mg} / \mathrm{kg} /$ day), to which we added praziquantel ( $50 \mathrm{mg} / \mathrm{kg} /$ day) for combination therapy. After 8 weeks, significant clinical improvement was noted, with complete resolution of both fever and hypotension. A liver magnetic resonance imaging scan performed after 7 months of continuous treatment revealed a $50 \%$ decrease in the size of the liver lesion, with residual satellite lesions (Figure 4). Praziquantel is not usually recommended for AE. However, because radical resection of the liver lesion was not possible, we opted for the addition of praziquantel to maximize potential protoscolicidal effects.

Experience with AE suggests that unresected disseminated disease should prompt lifelong antiparasitic treatment. Albendazole sulfoxide drug monitoring proved valuable for optimal dosing and managing toxicity. It was conducted monthly on serum drawn 4 hours post-morning dose (target 1-3 $\mu \mathrm{mol} / \mathrm{L})$ [8]. 


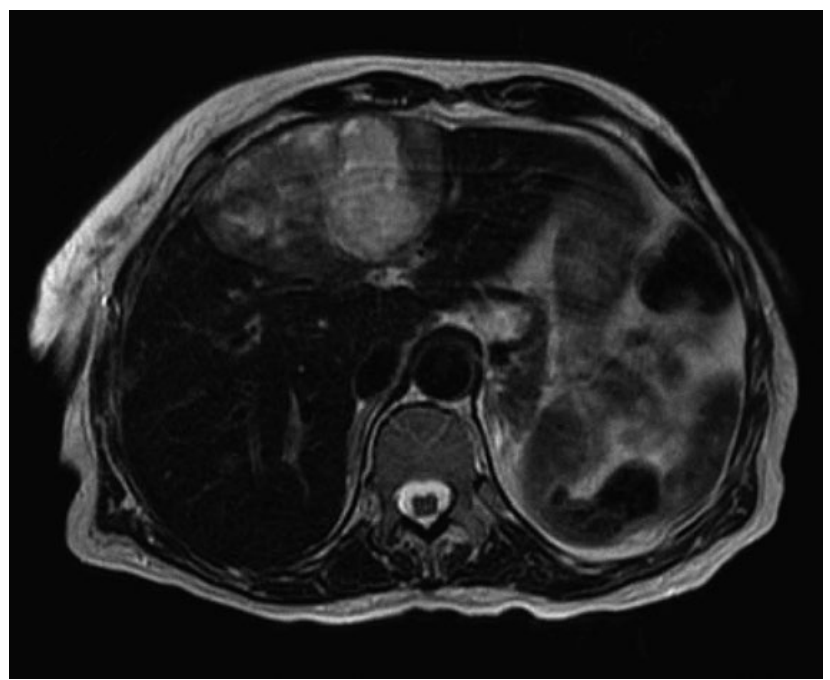

Figure 4. Axial T2-weighted magnetic resonance imaging scan of the liver, showing a large lesion occupying segments 4 , with residual satellite lesions 7 months after initiation of albendazole and praziquantel.

Partial liver resection for AE yields poor outcomes [8]. Liver allotransplantation has been performed in the context of complications such as cholangitis, secondary biliary cirrhosis, portal hypertension, hepatic hilar invasion, and Budd-Chiari syndrome, and has shown a 5-year survival rate of 66.7 to $71 \%$ [8]. In the current case, these complications were not observed and, after 7 months of combination chemotherapy, we documented a $50 \%$ decrease in the size of the liver lesion and resolution of many satellite lesions. Long-term follow-up with imaging and albendazole sulfoxide drug monitoring is planned.

The impact of immunosuppression on infections with metacestodes such as Echinococcus multilocularis is well described, often leading to rapidly-invasive progression and dissemination, reminiscent of malignancy [9]. This first human case of Versteria sp. infection, particularly in a kidney transplant recipient, is in keeping with these observations, including distant intrathoracic lymph node involvement. A documented normal abdominal CT scan 3 years prior to admission testifies to a relatively rapid disease progression. A Versteria infection reported in a captive Bornean orangutan in Milwaukee, Wisconsin, was also rapid, proved fatal, and involved the liver, lung, and spleen, although no immunosuppression was documented [7].

In our case, the patient presented with severe pulmonary and systemic symptoms, presumably attributed to extrahepatic dissemination of the infection. Along with the fever, the patient had multiple episodes of hypotension. We speculate that these episodes may be related to intermittent antigen release, resulting in a type I hypersensitivity reaction as described with E. granulosus and, less frequently, with E. multilocularis.

The clinical presentation, radiological findings, and inflammatory necrosis seen on histopathology were reminiscent of alveolar echinococcosis, but AE has never been described in humans or animals in the Eastern half of North America [10]. The histopathological appearance of the protoscolex and hooklets were not typical of E. multilocularis. Seen in less than $5 \%$ of human AE cases, the protoscolex of E.multilocularis is a circular, invaginated scolex with rostellar hooks of clearly-defined sizes and shapes (21-20 $\mu \mathrm{m}$ length and a ratio of the basis-length and bow-height of 1:3.4). By contrast, the metacestode of Versteria sp. (Figure 3) is a cysticercus-strobilocercus-like larvae whose scolex and rostellar hook dimensions are different from E. $m u l$ tilocularis [11]. Positive serology using crude antigens, as well as EgHF antigen for E. granulosus, likely reflects cross-reactivity between genera. Cross-reactivity was not observed with the E. multilocularis ELISAs, nor in the confirmatory Western blots.

Efforts to better characterize the definitive host of Versteria in North America have demonstrated at least 2 circulating parasite genetic lineages, representing separate introductions from Eurasia [12]. The definitive hosts of Versteria spp. are wild mustelids (eg, mink, ermine) and the intermediate hosts are rodents.

Sequence analysis of Versteria sp. found in feces of an ermine in Colorado, a mink in Oregon, the deceased index orangutan, and our patient all suggest a single lineage [12]. In the United States, Versteria sp. has also been reported in Wisconsin and Idaho. While both parasite genetic lineages have been found in the Northwest Territories of Canada, circulation of either has not been described anywhere else in the country [12]. We hypothesize that this immunocompromised patient acted as an accidental host.

Versteria sp. is a newly-recognized zoonosis and must be considered in the differential diagnosis of alveolar echinococcosis in immunocompromised patients from endemic areas. Unlike AE, combination medical therapy without surgery showed promising results. Distinguishing between $\mathrm{AE}$ and Versteria infection may thus have important prognostic significance.

\section{Supplementary Data}

Supplementary materials are available at Clinical Infectious Diseases online. Consisting of data provided by the authors to benefit the reader, the posted materials are not copyedited and are the sole responsibility of the authors, so questions or comments should be addressed to the corresponding author.

\section{Notes}

Acknowledgments. The authors thank Prof Dr Martin Fiedler and Yolanda Aebi from the Centre for Laboratory Medicine at the University Hospital of Bern for providing albendazole sulfoxide drug monitoring. They thank Bernadette Merkle, Ursula Kurath, and Xuan Lan Vu for performing laboratory diagnostic anaylses (serology and polymerase chain reaction). Finally, they thank Dr Christelle Thériault, who performed the liver biopsy by laparoscopy, and Drs Marie-Andrée Quirion and Emmanuel Maicas for the histopathology image.

Financial support. This work received no specific grant from any funding agency in the public, commercial, or not-for-profit sectors.

Potential conflicts of interest. All authors: No reported conflicts of interest. All authors have submitted the ICMJE Form for Disclosure of Potential Conflicts of Interest. Conflicts that the editors consider relevant to the content of the manuscript have been disclosed. 


\section{References}

1. Poretti D, Felleisen E, Grimm F, et al. Differential immunodiagnosis between cystic hydatid disease and other cross-reactive pathologies. Am J Trop Med Hyg 1999; 60:193-8.

2. Gottstein B, Jacquier P, Bresson-Hadni S, Eckert J. Improved primary immunodiagnosis of alveolar echinococcosis in humans by an enzyme-linked immunosorbent assay using the Em2plus antigen. J Clin Microbiol 1993 31:373-6.

3. Xiao N, Mamuti W, Yamasaki H, et al. Evaluation of use of recombinant Em18 and affinity-purified Em18 for serological differentiation of alveolar echinococcosis from cystic echinococcosis and other parasitic infections. J Clin Microbiol 2003; 41:3351-3.

4. Müller N, Frei E, Nuñez S, Gottstein B. Improved serodiagnosis of alveolar echinococcosis of humans using an in vitro-produced Echinococcus multilocularis antigen. Parasitology 2007; 134:879-88.

5. Müller N, Zimmermann V, Forster U, Bienz M, Gottstein B, Welle M. PCR-based detection of canine Leishmania infections in formalin-fixed and paraffin-embedded skin biopsies: elaboration of a protocol for quality assessment of the diagnostic amplification reaction. Vet Parasitol 2003; 114:223-9.
6. Trachsel D, Deplazes P, Mathis A. Identification of taeniid eggs in the faeces from carnivores based on multiplex PCR using targets in mitochondrial DNA. Parasitology 2007; 134:911-20.

7. Goldberg TL, Gendron-Fitzpatrick A, Deering KM, et al. Fatal metacestode infection in Bornean orangutan caused by unknown Versteria species. Emerg Infect Dis 2014; 20:109-13.

8. Vuitton DA, Azizi A, Richou C, et al. Current interventional strategy for the treatment of hepatic alveolar echinococcosis. Expert Rev Anti Infect Ther 2016; 14:1179-94.

9. Cooper AJR, Dholakia S, Holland CV, Friend PJ. Helminths in organ transplantation. Lancet Infect Dis 2017; 17:e166-76.

10. Massolo A, Liccioli S, Budke C, Klein C. Echinococcus multilocularis in North America: the great unknown. Parasite 2014; 21:1-13. Article number: 73.

11. Nakao M, Lavikainen A, Iwaki T, et al. Molecular phylogeny of the genus Taenia (Cestoda: Taeniidae): proposals for the resurrection of Hydatigera Lamarck, 1816 and the creation of a new genus Versteria. Int J Parasitol 2013; 43:427-37.

12. Lee LM, Wallace RS, Clyde VL, et al. Definitive hosts of Versteria tapeworms (Cestoda: Taeniidae) causing fatal infection in North America. Emerg Infect Dis 2016; 22:707-10. 